\title{
The Community Pharmacy Technician's Role in the Changing Pharmacy Practice Space
}

\author{
Brooke Taylor, PharmD, MS; Bella Mehta, PharmD, FAPhA
}

The Ohio State University College of Pharmacy

\begin{abstract}
Purpose: The practice of pharmacy and role of pharmacists has evolved over the decades but markedly since the introduction of the Affordable Care Act (ACA) in 2010. The ACA allowed patients to have increased access to community pharmacy services, such as medication therapy management, leading to an increase in the clinical services provided by pharmacists. This expansion of pharmacist's roles has led to pharmacists to feel an increase in workload which negatively impacts the time spent with patients. One way for this shift to occur without continuing to increase the pharmacist's workload is by using technicians as pharmacist extenders to take on more technical tasks.

Summary: The role of pharmacy technicians has been slow to expand from fear of public safety due to the lack of required education and training. Today, state requirements to practice as a pharmacy technician have become stricter with state requiring licensing, registration or certification. This increase in requirements as led to the expansion of pharmacy technician duties. Studies show that pharmacy technicians are able to perform technician accuracy checking, provide immunization and perform Clinical Laboratory Improvement Amendments (CLIA)-waived screenings. In addition to these duties, pharmacy technicians are being utilized in more novel ways such as collecting medication information in primary care and telepharmacy settings.

Conclusion: In order for pharmacy to continue to grow as a profession, pharmacists need to use pharmacy technicians as extenders. As pharmacy technicians begin to take on more of the technical duties, pharmacists are able to increase the time spent with patients.
\end{abstract}

Key Words: Pharmacy technicians, expanding roles, community pharmacy

\section{Introduction}

Community pharmacists are seeing an evolution in their role with the shift in healthcare focusing on quality of care, brought on by the Affordable Care Act. The act signed into law in 2010, helped to expand pharmacists' roles in coordinating care for patients and medication therapy management. While this was a great advancement for the profession of pharmacy, pharmacists feel an increased workload on a daily basis to provide their traditional dispensing services in addition to clinical services. According to the 2014 American Association of Colleges of Pharmacy's (AACP) National Pharmacists Workforce Survey, $66 \%$ of community pharmacists reported an increase or great increase in workload compared to a year ago. ${ }^{1}$ In addition to the increase in workload, $54 \%$ of community pharmacists stated that their workload negatively impacted the time spent in contact with patients and $35.5 \%$ of community pharmacists felt that their workload negatively impacted the quality of care provided to patients.

On average community pharmacists spend $75 \%$ of their time on patient care services associated with dispensing which includes calling providers, counseling on medications and preparing medications. ${ }^{1}$ The traditional role of a pharmacy technician is to aid the pharmacist in providing patient care services associated with dispensing that do not require professional judgement. The other $25 \%$ of the community pharmacist's day

Corresponding author: Bella Mehta, PharmD, FAPhA

Professor and Chair, Division of Pharmacy Practice and Science

The Ohio State University College of Pharmacy

500 W 12th Ave, Columbus OH 43210

Email: Mehta.6@osu.edu is spent on patient care services not associated with dispensing and business management at $13 \%$ and $12 \%$, respectively. Patient care services not associated with dispensing contain services billable by pharmacists such as completing comprehensive medication reviews, assessing medication needs, and adjusting medication dosages. Providing more patient care services not associated with dispensing can help close the gap between the actual dispensing cost and reimbursement received through the additional revenue. This shift in focus on clinical services to make revenue means that pharmacist's need to spend less time in the dispensing role and more time providing more clinical services. One way for this shift to occur without continuing to increase the pharmacist's workload is by using technicians as pharmacist extenders to take on more technical tasks. These technical tasks should include both traditional dispensing roles as well as novel nontraditional roles, such as vaccination administration and administration positions. To justify the expansion of pharmacy technician's role, pharmacists must recognize the history and development of pharmacy technician roles, understand pharmacy technician laws and regulation, and utilize technicians as pharmacist extenders.

\section{History of Pharmacy Technician Roles}

Pharmacy technicians have a long history in the pharmacy profession. The first formal pharmacy technician role dates back to approximately 1939 during World War 1 I. $^{2}$ Much like many pharmacy technicians today, technicians during this time were high school graduates without any formal training beyond employer-based training programs and their main duty was to assist the pharmacists in their daily tasks. For many years pharmacists were reluctant to increase the numbers of pharmacy technicians within pharmacies as they were hesitant 
to pay additional personnel and feared that they would take their careers. $^{3}$ In the 1960s and 1970s health-system pharmacists began to shift to more of a clinical role and automation became more common in pharmacies. ${ }^{2}$ These innovations led to more formalized roles of pharmacy technicians, particularly in the hospital setting.

Community pharmacists, on the other hand, were reluctant to the idea of utilizing pharmacy technicians out of concern for public safety. Due to this fear and increased number of pharmacy technicians, the U.S. Department of Health, Education and Welfare in 1968 recommended that pharmacy technicians have formal training in junior colleges and other institutions. While the creation of training guidelines and programs for health-system pharmacy technicians began by the mid-1970s, it wasn't until 1990s when community pharmacy technician roles become better defined, which in turn brought the development of national certifications and implementation of training for community pharmacy.

\section{Pharmacy Technician Rules and Regulations}

Today, the requirements and roles of community-based pharmacy technicians vary from state to state. Many states now require pharmacy technicians to become registered, certified or licensed but the requirements to obtain these designations is not uniform across the country. According to the Pharmacy Technician Certification Board (PTCB), registration is defined as "making a list of pharmacy technicians in the state or of being enrolled in an existing list" and licensure is defined as "the process by which an agency of government grants permission to an individual to engage in a given occupation upon recognizing that the applicant has attained the minimum competency necessary to ensure that the public health, safety, and welfare will be reasonably well protected". ${ }^{4}$ While these definitions help distinguish between registration and licensure nationally, the specific requirements and terminology to hold these designations still vary by state. Variability among states include roles such as registered technician, certified technicians, licensed technicians, as well as entry-level and advanced technicians. Many states that require pharmacy technicians to become registered have education or training requirements making it similar to a license. For example, in Indiana and Ohio all pharmacy technicians much be 18 years of age or older, obtain a high school diploma or GED, submit a criminal background check and complete an approved education program, however Indiana pharmacy technicians are termed to be "licensed technicians" while Ohio pharmacy technicians are considered "registered technicians" even though the requirements are the same. Currently, $73 \%$ of the United States require training of pharmacy technicians, however $35 \%$ of states require board approved training programs and only North Dakota requires an ASHP accredited program. $^{5}$

Certified pharmacy technicians are the only subset with a very defined set of requirements. In order for technicians to become nationally certified as a technician they must pass an examination approved by the Pharmacy Technician Certification Board (PTCB) or National Pharmacy Technician Association (NPTA) which attests the status of their education. In addition, pharmacy technicians must possess a high school diploma, disclose criminal and State Board of Pharmacy actions, complete an accredited education program and receive a passing score on the PTCB or NPTA examination. ${ }^{6,7}$

Table 1: Pharmacy Technician Requirements

\begin{tabular}{|l|c|}
\hline \multicolumn{1}{|c|}{ Required Status } & $\begin{array}{c}\text { Number of } \\
\text { States* }\end{array}$ \\
\hline Licensed & 11 \\
\hline Registered & 33 \\
\hline Certified & 24 \\
\hline Training & 38 \\
\hline *includes Washington D.C., Guam, and Puerto Rico \\
\hline
\end{tabular}

In addition to the variation in requirements to become a pharmacy technician there are variations in pharmacy technicians' roles. The traditional role common in all states include entering prescriptions into a pharmacy computer, counting medications, and affixing labels. ${ }^{5}$ As pharmacy expands the role of the pharmacy technician, they are given responsibilities in non-traditional roles such as accepting calledin prescriptions, checking the work of other technicians and administering immunizations. By allowing pharmacy technicians to take on these non-traditional roles, it will allow pharmacists to have more time to complete patient care services not associated with dispensing and allow for increased revenue.

Table 2: Pharmacy Technician Roles

Technician Role

Number of

States*

\begin{tabular}{|l|c|}
\hline Accept Called-in Prescriptions & 14 \\
\hline Administer Immunizations & 2 \\
\hline Call Physician for Refill Authorization & 41 \\
\hline Check Work of Other Technicians & 12 \\
\hline Compound Medication for Dispensing & 52 \\
\hline Enter prescriptions into Pharmacy Computer & 53 \\
\hline Transfer Prescriptions & 12 \\
\hline
\end{tabular}

*includes Washington D.C., Guam, and Puerto Rico

\section{Pharmacy Technicians as Pharmacist Extenders}

In order for the scope of pharmacists' practice to continue to grow, pharmacists should utilize their pharmacy technicians as extenders. As community-based pharmacists develop new roles, they are expected to do more with less time by providing an increase number of clinical services while performing traditional dispensing for more patients. By utilizing pharmacy technicians to their full potential, pharmacists can increase time spent with patients and conducting clinical services. The 
term technicians as pharmacist extenders means that pharmacy technicians are used based on their full scope of practice to help pharmacists reach more patients, improve clinical outcomes and increase revenue. The full scope of pharmacy technicians includes the traditional roles of support for dispensing but also includes non-traditional roles of administering immunization, technician accuracy checking, and facilitation of Clinical Laboratory Improvement Amendments (CLIA)-waived screenings. Studies have shown that pharmacy technicians are capable of performing these non-traditional tasks by being just as safe and accurate as pharmacists and it allows pharmacist to spend more time in the clinical role. ${ }^{8-11}$

Pharmacy technician provided immunizations and technician accuracy checking are two areas that pharmacy technicians have recently been given more responsibility to complete. Currently only three states, Rhode Island, Idaho and Utah, allow pharmacy technicians to administer all United States Centers for Disease Control and Prevention (CDC) recommended vaccinations to patients. In these states, technicians must complete a 6-hour training program from the Accreditation Council for Pharmacy Education (ACPE) that contains 2 hours of home study material and a 4-hour live training or American Pharmacists Association (APhA) Pharmacy-based Immunization Delivery training. In addition to the training, technicians must be PCTB or NPTA and basic life support (BLS) certified. While there have been no randomized controlled trials conducted on technician administered vaccinations, Idaho collected anecdotal data from the 6-month pilot period before it became a law. During this 6-month period, 25 technicians administered 935 vaccines to patients that were 6 years or older and no adverse events were reported. ${ }^{8}$ Currently there are no studies showing the direct benefit of pharmacy technicians administering vaccinations, however the profession of pharmacy can extrapolate anecdotal data from physician and other medical practice models. In these practice models, physicians often delegate vaccination administration to medical assistants and nurses whom have similar or less training as pharmacy technicians. While more studies need to be conducted to show the true economic and clinical benefit of pharmacy technicians administering vaccinations, with millions of immunizations provided to patients each year the pharmacists' time saved is the largest benefit.

Technician accuracy checking is another role that is becoming more common as part of pharmacy technician's full scope of practice. Technician accuracy checking (TAC) is defined as utilizing technology to check the work of someone else, an automated dispensing system or other technology assisted filling equipment. An example of accuracy checking includes having a technician ensure that the current product and quantity was dispensed by an automated dispensing machine. Currently, 20 states allow TAC either through a pilot program or is board approved but 10 states only allow it in the outpatient setting. In the inpatient setting, there have been 11 studies on TAC in which all show that technicians were just as accurate, if not more accurate, than pharmacists at checking the accuracy of dispensed medication. In the community setting four studies have been conducted and found similar results to the inpatient studies; that technicians are just as accurate as pharmacists at accuracy checking. ${ }^{9-10}$ In a study conducted by the University of Wisconsin researchers found that technicians are $99.95 \%$ accurate compared to pharmacist whom are $99.74 \%$ accurate. ${ }^{9}$ In another study that looked at overall errors as well as administrative and patient safety errors found no statistically significant difference between pharmacists and pharmacy technicians. ${ }^{10}$ In addition to technicians being equally accurate as pharmacists, two studies showed that by allowing technicians to check medications it allowed the pharmacists to have more time, equivalent to approximately 23 days a year, to complete other direct patient care tasks in the day. The increase in time in direct patient care allowed the pharmacist to spend significantly more time providing counseling to patients on medications and determining proper drug utilization. $^{9}$

\section{Novel Technician Duties}

In pharmacy, there has been an increase in the number of point of care testing (POCT) services provided to patients. CLIAwaived POCTs, which are defined as simple tests with a low risk for an incorrect result, are quick tests that may be performed by pharmacists and pharmacy technicians. Some common POCT performed by pharmacists and pharmacy technicians include cholesterol, blood glucose and International Normalized Ratio (INR) testing. Patients want quick and easy clinical services, and many pharmacies and ambulatory care clinics have shorter wait times than a traditional emergency room or acute care clinic leading to increased demand for POCTs and services. One practice model that would utilize pharmacy technicians would be having the technician set up appointments for patients, gather patient information when rooming patients for appointments and perform the CLIAwaived point of care test. This model would allow the pharmacist to see an increase number of patients since the pharmacy technician would be obtaining the laboratory value leaving the clinical decision making to the pharmacist. Some common CLIA-waived point of care tests performed by pharmacies include blood glucose, cholesterol, influenza, group B streptococcus, human immunodeficiency virus (HIV) and human papillomavirus (HPV) tests. As the scope of point of care testing completed in a community pharmacy and ambulatory care setting grows, the more pharmacists will need to rely on their pharmacy technicians.

Another novel role for pharmacy technicians would be when patients are admitted to an urgent care clinic or present to a community pharmacy either in person or virtually though telehealth to obtain patient information such as a medication history. The practice model would be similar to the point of care testing and would mirror that of a medical assistant or nurse and physician or physician assistant during a doctor's appointment. The pharmacy technician would collect all 
background information then the pharmacist would see or talk to the patient. Again, this model would allow the pharmacist to focus on the medication related problems and clinical recommendations by having all needed information collected before seeing the patient. In rural areas, telepharmacy could be used to allow the pharmacist to access a larger patient population and allow patients to stay close to home for their care if done virtually. The practice model would work the same as in person appointments but would be done through a computer or phone.

Point of care testing and medication management services can provide additional revenue to ambulatory care clinics and community pharmacies when provided in person or virtually. At many community pharmacies, point of care testing is an out of pocket service for patients providing direct revenue to the pharmacy and the cost of these services vary by location. In ambulatory care clinics, pharmacists can bill incident-to a physician for services as a source of revenue. The reimbursement for pharmacy services billed incident-to a physician depends largely on the complexity of the appointment but could range from approximately $\$ 20$ to $\$ 100$ and depending on state regulations telepharmacy services may be billed similarly to in-person pharmacy services with similar reimbursement. The benefit of utilizing pharmacy technicians to help provide these services is the amount of time saved to pharmacists. In a study coordinated by the Pittsburgh Regional Health Initiative (PRHI), pharmacy technicians performed tasks such as obtaining patient medication, retrieving laboratory information from the patient's chart and gathering patient education materials which allowed for a $40.5 \%$ increase in the number of CMRs completed by pharmacists. ${ }^{11}$

Lastly, pharmacy technicians could be utilized in management roles needed to ensure the pharmacy runs efficiently such as inventory management, $340 \mathrm{~B}$ coordination, informatics, and billing and finance integrity. These roles are very important to make sure that the pharmacy has sufficient inventory, reimbursed properly, and meeting compliance standards of the many regulatory bodies; and can take a lot of time to be done properly. While some health-systems utilize their pharmacy technicians in management roles, there is a great opportunity in the outpatient space. As frontline staff, pharmacy technicians are knowledgeable in possible workflow enhancements that need to be made, inventory management strategies and education and training of other technicians. Utilizing pharmacy technicians in these roles, once again allows pharmacist to spend more time performing clinical duties.

\section{Conclusion}

As the world of healthcare continues to evolve so does the profession of pharmacy. While stagnant for many years, the role of the pharmacy technician has begun to evolve since 1939 when pharmacy technicians had minimal assistant duties. This movement is in large part due to more regulated training and registration or licensure requirements to be a pharmacy technician. As pharmacy technician's roles begin to expand, registration or licensure requirements need to become more standardized. This standardization will not only help pharmacist feel more comfort allowing technician to take on more tasks but allow boards of pharmacy more oversight of technicians.

In order to be able to provide the services necessary to create a sustainable profession, pharmacy cannot get complacent and must utilize our pharmacy counterparts. By allowing pharmacy technicians to take on non-traditional roles such as administering immunizations, technician accuracy checking and point of care testing it allows pharmacists to provide more clinical services and increase the time spent with patients. While more studies need to be done to investigate the direct economic and clinical impact of these non-traditional roles of pharmacy technicians, the impact of pharmacists provided clinical services has shown a direct correlation to better health outcomes, increased patient satisfaction and positive economic impact.

\section{Conflicts of Interest: None}

\section{References}

1. Gaither CA, et al. Final report of the 2014 National Sample Survey of the Pharmacist Workforce to determine contemporary demographic, practice characteristics and quality of work-life. AACP. 2015 Apr 8.

2. McCarthy R. Pharmacy Technicians. American Institute of the History of Pharmacy. 2016. Available from:https://aihp.org/wpcontent/uploads/2018/08/4-PharmacyTechnicians.pdf

3. CCl Training Center [Internet]. Dallas (TX): CCl Training Center. Pharmacy Technicians: The Past, Present and Future; 2015 Dec 26 [cited 2019 May 10]. Available from:

https://www.ccitraining.edu/blog/pharmacytechnicians-the-past-present-and-future/

4. Pharmacy Technician Certification Board [Internet]. Washington (DC): Pharmacy Technician Certification Board. How can I obtain a state license?; 2019 Nov 23[cited 2019 May 12]. Available from: https://www.ptcb.org/resources/cpht-toolkit/stateregulations\#.XUIpyS2ZNQI

5. Survey of Pharmacy Law. Mount Prospect (IL): National Association of Boards of Pharmacy; 2017.

6. Pharmacy Technician Certification Board [Internet]. Washington (DC): Pharmacy Technician Certification Board. Credentialing Guidelines and Requirements; [updated 2020; cited 2019 May 13]. Available from: https://www.ptcb.org/guidebook/ptcb-certifiedpharmacy-technician-cpht-program 
7. National Pharmacy Technician Association [Internet]. Houston (TX): National Pharmacy Technician Association. The ExCPT Exam; 2010 [cited 12 May 2019]. Available from:

http://www.pharmacytechnician.org/excpt/

8. McKeirnan KC, et al. Transforming pharmacy practice: Advancing the role of technicians. Pharmacy Today. 2018 Jun.

9. Miller RF, et al. Evaluation of Community Pharmacy Tech-Check-Tech as a Strategy for Practice Advancement. J Am Pharm Assoc. 2018 Nov.

10. Andreski $\mathrm{M}$, et al. The lowa new practice model: Advancing technician roles to increase pharmacists' time to provide patient care services. J Am Pharm Assoc. 2018 May.

11. Fera $\mathrm{T}$, et al. Clinical support role for a pharmacy technician within a primary care resource center. Am J Health Syst Pharm. 2018 Feb 1;75(3):139-144. 